\title{
Pharmacoeconomics of NSAIDs: Beyond Bleeds
}

\section{OBJECTIVE:}

To determine the marginal effect of including a minor, yet common, gastrointestinal (GI) side effect in a cost effectiveness analysis of NSAID therapy.

\section{DESIGN:}

Base economic model of cost effectiveness analysis (CEA) developed using data from a randomized controlled trial of two formulations of an NSAID therapy (conventional versus extended release etodolac) for the treatment of osteoarthritis (OA) of the knee.

\section{SETTING:}

Outpatient.

\section{PARTICIPANTS:}

225 patients with osteoarthritis of the knee.

\section{INTERVENTIONS:}

Patients were randomized to receive either

\author{
C. Daniel Mullins, Lisa Stockwell Morris, \\ Eleanor M. Perfetto, Shelby D. Ogilvie
}

conventional or extended release etodolac with primary endpoints evaluated at four weeks. Cost effectiveness was calculated from a managed care perspective as dollars spent per OA patient treated, focusing on the marginal dollar value of reduced side effects. Costs included in the analysis were initial ( $\$ 64$ or $\$ 71$ ) and subsequent (\$64) prescriptions for NSAID therapy, pharmacological treatment of side effects $(\$ 79)$, and physician office visits $(\$ 50)$.

\section{MAIN OUTCOME MEASURES:}

Percent reduction in dyspepsia, average cost per OA patient treated.

\section{RESULTS:}

Reduced instances of dyspepsia, a common side effect of NSAID therapy, from $20 \%$ to $8 \%$ resulted in an estimated marginal cost savings to the insurer of $\$ 14$ per patient switched from conventional to extended-release etodolac. In addition, there were cost savings to the patient in terms of reduced out-of-pocket expenses. A more favorable side-effect profile also may assist in improving patient compliance.

\section{CONCLUSION:}

The marginal effect of including common Gl side effects in a CEA of NSAIDs may have important economic implications for managed care providers. While ulceration and bleeds are the most serious and costly side effects associated with NSAID therapy, it is important to consider other positive and negative outcomes associated with therapy when performing a pharma. coeconomic evaluation.

\section{KEY WORDS:}

NSAID; Pharmacoeconomics; Outcomes; Osteoarthritis; Dyspepsia.

J Managed Care Pharm 1997; 3: 425-30.
Osteoarthritis (OA) is a common condition affecting weight-bearing joints of the axial and peripheral skeleton, primarily the hips, knees, hands, and feet. 'Signs and symptoms include pain, inflammation, limitation of motion, and deformity of the joint. OA is highly prevalent, and is the second leading cause of severe chronic disability among adults. ${ }^{2}$ Approximately $12 \%$ of the U.S. population between the ages of 25 and 74 have signs and symptoms of $\mathrm{OA}$. The prevalence of moderate and severe cases appears to increase with age: ${ }^{3.4}$ about $50 \%$ of adults between 65 and 74.years of age suffer from moderate to severe OA of the hip, and $33 \%$ have mild to moderate OA of the knee. ${ }^{3,4}$

NSAIDs are central to the long-term pharmacologic treatment of patients with rheumatoid arthritis, $O A$, and other painful musculoskeletal conditions. NSAIDs are widely prescribed for the treatment of $O A$, given their proven efficacy and safety in relieving the pain and inflam-

C. DANIEl MUluINS, Ph.D., Assistant Professor, Department of Pharmacy Practice and Science, University of Maryland at Baltimore, Baltimore, MD. LISA STOCKWELL MORRIS, Ph.D., R.Ph., Director, Disease, Treatment, and Outcomes Information Services, IMS America. (At the time this article was written, Dr. Morris was Associate Director, Health Outcomes Assessment, Wyeth-Ayerst Research, Radnor, PA.) ELEANOR M. PERFETTO, Ph.D., R.Ph., CEO of MEDTAP Systems, LLC. (At the time this article was written Dr. Perfetto was Director, Health Outcomes Assessment, Wyeth-Ayerst Research, Radnor, PA.) SHELBY D. OGILVIE, R.Ph., doctoral candidate, Department of Pharmacy Practice and Science, University of Maryland at Baltimore, Baltimore, MD.

AUTHOR CORRESPONDENCE: Daniel Mullins, Ph.D., Pharmacy Practice and Science; 100 Penn St., Room 255, Baltimore, MD 21201.

ACKNOWLEDGEMENT: Partial funding for this research was provided by Wyeth-Ayerst Research

Copyright (C) 1997, Academy of Managed Care Pharmacy, Inc. All rights reserved: 
mation associated with this condition. World-wide, approximately 30 million people consume NSAIDs on a regular basis. ${ }^{5}$ Within the U.S., more than 99 million prescriptions for NSAIDs are filled annually, ${ }^{6}$ and six of the top 100 drugs dispensed at community pharmacies in 1994 were NSAIDs. ${ }^{7}$ In addition, different NSAID formulations now are available as OTC products in the U.S., significantly increasing the number of people exposed to this class of drugs.

\section{SAFETY AND EFFICACY OF NSAIDS}

Numerous clinical studies have demonstrated the safety and efficacy of NSAIDs in the treatment of arthritic conditions. A range of NSAIDs with comparable treatment effects is available to physicians and their patients. All of the agents in this class produce similar therapeutic benefits and have similar side-effect profiles, and no particular NSAID has demonstrated uniform superiority. ${ }^{8,910,11,12}$ However, some NSAIDs require frequent dosing and others can be administered once a day, which may influence patient compliance, affect convenience, and/or influence cost. An NSAID's safety profile, dosing frequency, and cost are three influential factors in prescribing or formulary decision making in the selection of which NSAID is likely to provide optimal patient care.

NSAIDs are generally considered safe. However, their extensive use has prompted concern related to effects of NSAIDs on the upper gastrointestinal (GI) tract, especially since approximately $25 \%$ of NSAID users develop GI-related side effects. ${ }^{13}$ Serious side effects such as hemorrhage and perforation are rare, but a substantial proportion of patients treated with NSAIDs develop more common, noncatastrophic events such as dyspepsia, nausea, and vomiting. ${ }^{14}$ However, it appears that individual NSAIDs differ in their potential to cause GI toxicity. ${ }^{15}$ In general, newer NSAIDs have demonstrated better safety profiles than aspirin. ${ }^{16}$ For example, etodolac has been compared with a number of other NSAIDs in endoscopic studies, which demonstrated that it produces fewer GI-related side effects than aspirin, naproxen, ibuprofen, or indomethacin, while its effects on the GI tract are comparable to those of placebo. ${ }^{17,18,19.20 .21}$ In addition, two studies have shown that etodolac ER produces fewer GI-related adverse effects than other NSAIDs. The first study (single-blind, openlabel) revealed significantly less GI blood loss in both groups treated with etodolac ER (600 mg daily and $1200 \mathrm{mg}$ daily) when compared to the group receiving naproxen $(500 \mathrm{mg}$ twice daily). ${ }^{22}$ These results may be questioned due to the fact that they are based on a single-blind, open-label study and not a traditional clinical trial; however, the observer performing the blood loss analysis remained blinded. In another study, a randomized, double-blind trial compared the safety and efficacy of etodolac ER and tenoxicam in elderly patients with $\mathrm{OA}$ of the hip and knee. Endoscopic findings revealed more minor lesions in the stomach mucosa among those patients receiving tenoxicam ( $20 \mathrm{mg}$ twice daily) compared to those treated with etodolac ER ( $600 \mathrm{mg}$ daily). ${ }^{23} \mathrm{It}$ is possible to have micro blood loss without the existence of GI symptoms. This relationship was not examined in the clinical trial.

\section{COST CONSIDERATIONS OF NSAID-RELATED GASTROINTESTINAL TOXICITY}

Gastrointestinal side effects can produce suboptimal patient outcomes as well as contribute to increased treatment costs. Expenditures for treating GI side effects alone have been shown to comprise $46 \%$ of total expenditures for arthritis care. ${ }^{13} \mathrm{Al}-$. though a small proportion of patients hospitalized for GI complications accounted for a majority of total expenditures, most patients with NSAID-associated GI side effects experienced gastritis or duodenitis and abdominal pain that required treatment but not hospitalization. It is likely that many of these patients self-treated such symptoms with OTC medications. Nonetheless, the aggregate costs accounted for nearly a quarter of total expenditures for treating GI side effects. ${ }^{13}$

One treatment option available to decrease the risk of GI side effects is the use of an analgesic such as acetaminophen that produces few or no GI side effects; however, it lacks the anti-inflammatory property of NSAIDs. Another is to use misoprostol for prophylaxis against NSAID-induced GI tract bleeding. In a cost-effectiveness analysis, Edelson and colleagues concluded that using misoprostol for primary prevention would be very costly for the general NSAID-using population ( $\$ 667,400$ per year of life saved) ${ }^{24}$ However, its incremental cost-effectiveness ratio was much more attractive $(<\$ 40,000$ per year of life saved) when used for secondary prevention in patients with a previous GI event.

A large pharmacoepidemiologic study recently documented that approximately $15 \%$ of patients takking NSAIDs reported experiencing an NSAID-related GI side effect over a 2.5year period. ${ }^{25}$ While most of these patients experienced no other event, approximately $12 \%$ stopped or changed NSAID therapy and almost $40 \%$ had GI medications added to their drug regimen. However, NSAIDs with various GI side-effect profiles were not analyzed in the study, and the authors acknowledged that the conclusions based on NSAIDs as a whole may not be applicable to individual agents. ${ }^{25}$ In light of their widespread use, side-effect profile, and the high costs associated with prophylaxis against NSAID-induced GI toxicity, it is beneficial to explore the value of NSAIDs that inherently produce a lower rate of GI side effects. The objective of this research was to determine the marginal effect of including a minor, yet common, gastrointestinal side effect in a costeffectiveness analysis of NSAID therapy.

\section{METHODS}

A decision analytic model of NSAID therapy based on data for $225 \mathrm{OA}$ patients from a randomized, clinical trial (RCT) was used to determine the marginal effect of including 


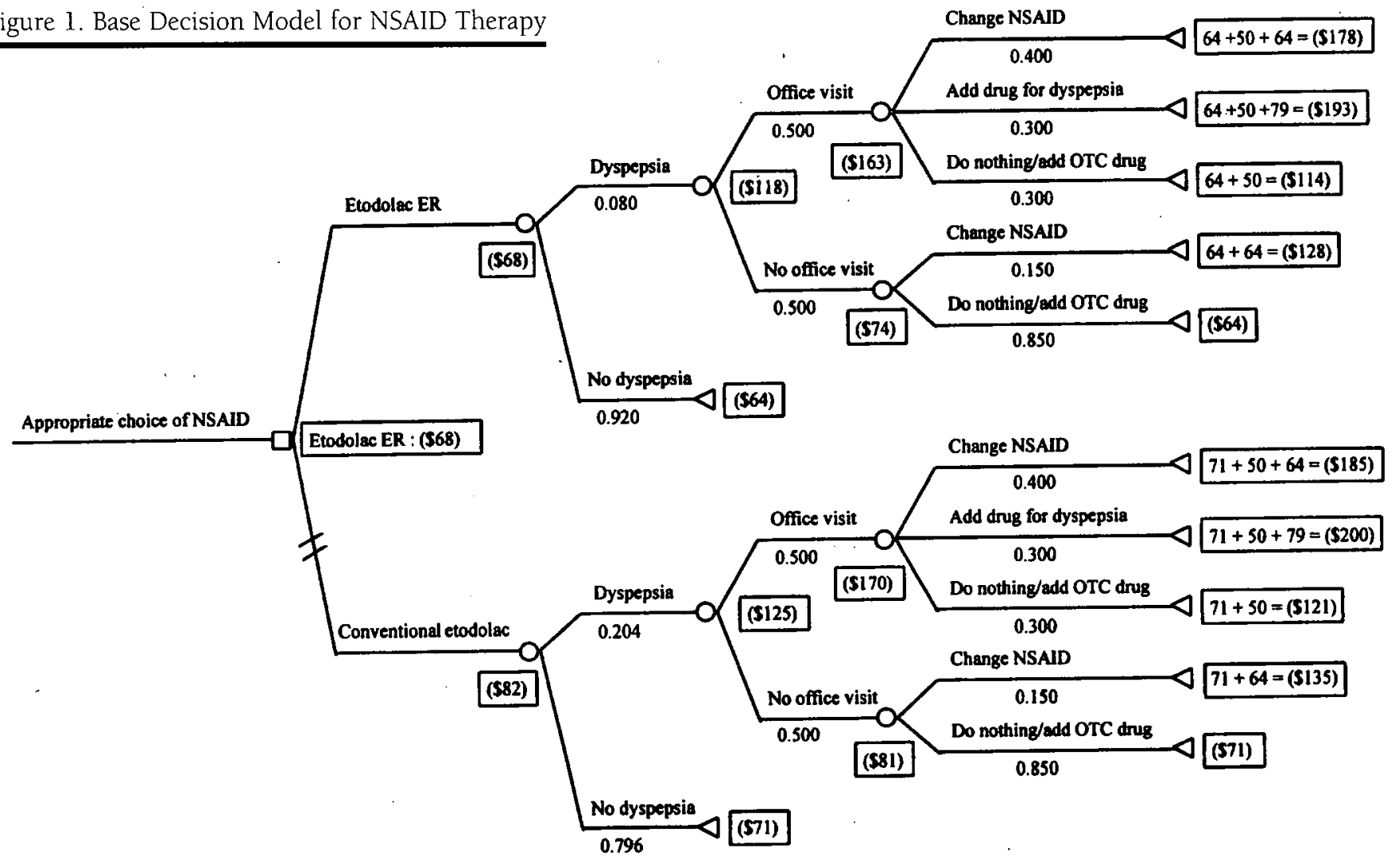

more common GI side effects in a cost-effectiveness analysis. Although the clinical trial (Protocol 654-D-323-US) employed three study arms, this CEA examined only etodolac $1200 \mathrm{mg}$ $\mathrm{ER}$ and etodolac $400 \mathrm{mg}$. TID. The etodolac $800 \mathrm{mg}$ ER arm was deleted for ease of presentation and because $1200 \mathrm{mg}$ ER contains the same amount of active ingredient as $400 \mathrm{mg}$ TID. Furthermore, the results are similar for $800 \mathrm{mg}$ ER and 1200 $\mathrm{mg}$ ER. This article examines the primary end point at four weeks, although the double-blind clinical trial continued for 24 weeks.

The efficacy and safety of a new, extended release formulation of etodolac, etodolac ER, has been documented in a number of clinical trials. ${ }^{23,26.27}$ Etodolac ER has been shown to be as efficacious as the conventional form of etodolac in terms of symptom relief, but it produces a significantly lower incidence of dyspepsia (8\% versus 20\%). ${ }^{27}$ Etodolac ER is similar to the conventional form regarding other side effects. Thus, the probability of dyspepsia, and the potential ensuing consequences such as additional office visits, additional prescriptions, and purchase of OTC products, should be included in a decision model (or decision tree) that examines the costeffectiveness of NSAID therapy (see Figure 1).

\section{Decision Analysis}

The marginal effect of reduced side effects can be represented by a decision tree which includes the probability of experienc- ing the side effects, along with the subsequent actions and their corresponding costs. In the case of side effects associated with NSAID treatment, one can examine the marginal costs of treating GI complications. When examining the marginal effect, it is only necessary to include those effects that differ across treatment alternatives. For this reason, the authors have not included the initial office visits since this occurs in all cases. This simplifies the presentation by focusing solely on those events that have differential health outcomes and economic consequences.

The decision tree presented here can be used to determine whether conventional etodolac or etodolac ER would be the more cost-effective NSAID therapy for a managed care institution. The model makes the following assumptions about the costs to a managed care organization: (1) the cost of a physician office visit is $\$ 50$; (2) the prescription cost for one month's supply of an NSAID other than etodolac or etodolac ER is $\$ 64$ based on average wholesale price (AWP) less 15\% for the top five products, (3) the prescription cost for one month's supply of an $\mathrm{H}_{2}$-receptor antagonist or other pharmaceutical agent for treatment of dyspepsia is $\$ 79$; (based on AWP less 15\% for the top five products) and (4) there is no drug cost to the managed care provider when an OTC medicine is prescribed. In addition, the prescription cost for conventional etodolac ( $\$ 71)$ is based on AWP less 15\% for one month's supply of Lodine ${ }^{\circledR} 400$, while the cost for etodolac 
ER ( $\$ 64)$ is based on AWP less 15\% for one month's supply of Lodine XL® ${ }^{\circledR} 400$. Prices for a one-month supply of NSAID or anti-ulcer therapy is based on a $15 \%$ discount off the average wholesale price of the most widely prescribed NSAIDs at therapeutically equivalent dosages, as reported in the Red Book. The selection of the top five products for each category was based on the top 100 drugs of $1995 .{ }^{28}$

The left-most branch of the tree represents the decision to be made regarding appropriate selection of NSAID therapy from a cost-effectiveness standpoint. Regardless of which formulation is chosen, there is the possibility of a patient experiencing dyspepsia or not experiencing dyspepsia. The probabilities are based on the results of clinical trial data from one study (Protocol 654-D-323-US) and are presented underneath each branch. Patients experiencing dyspepsia may elect to visit their physician. Patients with dyspepsia who visit a physician may receive a prescription for a different NSAID or they may be treated for their dyspepsia, either with a prescription product or an OTC medicine. Estimates of the probability of visiting a physician and receiving a prescription for a different NSAID are based on national estimates published by Walker, Chan, and Yood. ${ }^{29}$ Those patients who do not visit the physician may still have their NSAID therapy changed over the phone, although it is assumed that this would happen much less frequently than when an office visit occurs $(0.15$ vs 0.40 ). It is possible for a physician to order a prescription drug treatment for dyspepsia over the phone, but this possibility is not included in the analysis for simplicity of illustration. The true probability for switching a patient's NSAID therapy over the phone is unknown, so a sensitivity analysis was performed.

The "do nothing" branch includes a variety of options in which the physician does not recommend changing or adding an additional prescription drug. This branch covers patient self care, including cases in which a physician recommends an OTC product. The economic implication is that a managed care provider has no additional payment for any options included under "do nothing."

The determination of the expected patient cost, which is the average cost to a managed care provider that covers a large number of $\mathrm{OA}$ patients, requires 'rolling back' the tree. The expected cost at any branch is simply the probability of being on that branch multipled by the associated total costs... For example, a patient who experiences dyspepsia and goes for: an office visit has a 0.4 probability of being prescribed a new NSAID. Costs to the managed care provider include either $\$ 71$ (conventional etodolac) or $\$ 64$ (etodolac ER) for the initial NSAID, $\$ 50$ for the office visit, and $\$ 64$ for the newly prescribed NSAID, for a total cost of either \$185 (conventional etodolac) or $\$ 178$ (etodolac ER). Similarly, the probability that such a patient would remain on the initial NSAID and be given a prescription for an $\mathrm{H}_{2}$-receptor antagonist or other drug to treat dyspepsia is 0.3 , with a total cost of $\$ 200$ (conventional etodolac) or $\$ 193$ (etodolac ER). The total cost to the managed care provider when the patient purchases an OTC_product for dyspepsia is only $\$ 121$ (conventional etodolac) or $\$ 114$ (etodolac ER), since the managed care provider only pays for the initial NSAID and the office visit, not the OTC product. Since the patient receives an OTC with a probability of 0.3 in this scenario, the expected cost for a patient treated with etodolac ER who experiences dyspepsia and goes for an office visit is $(0.4 \times \$ 178)+(0.3 \times \$ 193)+$ $(0.3 \times \$ 114)=\$ 163$, as shown in the decision tree. This process is repeated from right to left for all branches to arrive at the expected costs of treatment for both treatment arms.

\section{RESULTS}

\section{Patients}

During the four-week, double-blind segment of the RCT, a total of 113 patients received conventional Lodine ${ }^{\circledR}$ (etodolac $400 \mathrm{mg}$ TID), while 112 patients received Lodine XL® (etodolac ER $1200 \mathrm{mg}$ daily), both manufactured by WyethAyerst Laboratories. The vast majority of the patients were white (88\% for conventional etodolac; $89 \%$ for etodolac ER) and female ( $73 \%$ for conventional etodolac; $77 \%$ for etodolac ER).

\section{Drug Effectiveness}

Patient efficacy data were analyzed for two patient populations: intention-to-treat (ITT) and valid for efficacy (VFE). The four key efficacy variables were investigator's overall evaluation, patient's overall evaluation, overall pain intensity, and walking pain. The level of statistical significance was set at $\mathrm{p} \leq 0.05$. For the two groups compared in the ITT analysis at the primary end point at four weeks, there were no statistically significant differences in three of the four key efficacy measures. For the patient's overall evaluation, there was a statistically significant higher mean improvement in the $400 \mathrm{mg}$ TID. group than in the ER $1200 \mathrm{mg}$ group. For the VFE analysis at . the primary end point, there were no statistically significant differences in any of the key efficacy variables. In terms of emergent study events, there were statistically significantly fewer events among the ER $1200 \mathrm{mg}$ group than among the $400 \mathrm{mg}$ TID group for both dyspepsia and the aggregate measure for the digestive system. As reflected in Figure 1, the reported rates of dyspepsia were 0.204 and 0.08 , respectively for conventional and extended release etodolac.

\section{Therapy Cost}

From the insurer's perspective, the total cost of therapy includes not only the cost of the initial treatment but also any subsequent office visits as well as any new prescriptions related to side effects of the prescribed NSAID. New prescriptions would include changes to a different NSAID as well as additional medication to treat dyspepsia. The cost for items in each branch of the decision tree then is the total cost shown at the right-most branch, multiplied by the corresponding probabilities. When this procedure is' implemented consecutively 
from the right-most branches to the initial decision, the total cost of therapies for conventional etodolac and etodolac ER, respectively, are $\$ 82$ and $\$ 68$.

\section{Cost Effectiveness}

The marginal effect on the cost effectiveness of NSAIDs that provide similar safety profiles (except with reduced dyspepsia) is calculated by comparing the expected cost of therapies across the two main branches. The marginal cost difference between conventional etodolac and etodolac ER is \$14 per patient switched from conventional to extended release etodolac. This $\$ 14$ savings represents a one-time projected savings during the month following the initial prescription; however, additional savings may occur if improved compliance leads to improved therapeutic outcomes. Longitudinal follow-up of patient outcomes was not captured in the clinical triali, so additional long-term savings cannot be captured.

\section{Sensitivity Analysis}

Sensitivity analysis was performed by varying the probabilities associated with physician prescribing patterns for patients who had an office visit related to dyspepsia. Specifically, the probabilities of changing the NSAID or adding a prescription drug for dyspepsia were varied, from a low of 0.20 and 0.20 respectively, to a high of 0.5 and 0.4 . The perpatient savings to the managed care provider ranged from $\$ 12$ to $\$ 15$ under the revised assumptions. Next, the probability that a patient experiencing dyspepsia would have an office visit was revised to a low estimate of $35 \%$. This reduced the cost savings per patient to $\$ 12$. Although the probabilities of experiencing dyspepsia for etodolac ER and conventional etodolac were based on a clinical trial, a larger group of patients was available by combining data from several clinical trials. When the entire population of patients examined for FDA approval were considered, the reported probabilities of dyspepsia were $6.5 \%$ and $16.6 \%$ for etodolac ER and conventional etodolac, respectively. Using these probabilities in the model in place of the ones from the single clinical trial yielded cost savings of $\$ 12$ per patient.

Finally, a worst-case scenario was performed in which multiple assumptions were altered simultaneously. This final analysis assumed rates of dyspepsia of $6.5 \%$ and $16.6 \%$ for the etodolac ER and conventional groups, respectively. Furthermore, the probability of an office visit given that a patient experiences dyspepsia was assumed to be 0.35 . The probabilities of a change in NSAID or the addition of a prescription drug for dyspepsia, given an office visit, were each set at 0.20 . It was further assumed that no patients received a prescription drug for dyspepsia or had their NSAID changed without an office visit. When all of these assumptions were entered into the model simultaneously, savings to the managed care provider were estimated at $\$ 10$ per patient.

\section{DISCUSSION}

A CEA should include both positive outcomes, measured by efficacy and patient well being, and negative ones, such as adverse events and other factors that negatively influence patient quality of life (i.e., dyspepsia, in this study). The decision analysis presented in this article demonstrates the marginal effect of including a common GI side effect, dyspepsia. The limited number of comparative drug therapies in the clinical trial on which this analysis was based does not allow for comparisons across alternative NSAID therapies. Also, because the two formulations of etodolac were previously demonstrated to be similar along all other measures, only the reduction of dyspepsia was considered in performing the analysis. While additional comparative studies incorporating a wider scope would be beneficial, the current analysis demonstrates the important effect that common GI side effects can have on the cost effectiveness analysis of NSAID therapy.

These results highlight the importance of including all relevant factors in a cost-effectiveness analysis. In an analysis of NSAID therapies, the treatment costs for bleeds associated with NSAID use represent large expenditures, but there is a low rate of occurrence of bleeds. On the contrary, the rate of dyspepsia is relatively high, although the associated costs of treatment are low on a per-patient basis. Since managed care providers make formulary decisions at the population level, the aggregate costs of treatment are important. Thus, those side effects with low per-patient costs that occur in a large proportion of the population are important factors for pharmacoeconomic evaluation.

The decision analytic model presented in this analysis only considers those costs borne by the insurer or managed care provider. Also to be considered are cost savings to the patient in terms of reduced out-of-pocket expenses (e.g., OTC medications, travel to the physician office) and a more favorable side-effect profile, along with the simplicity of the once-daily dosing of etodolac ER, that may improve patient compliance.

\section{CONCLUSION}

Examination of common GI side effects should enhance, not replace, the rare event of NSAID-induced ulceration and bleeding in a pharmacoeconomic model. When comparing two NSAIDs with similar efficacy and safety profiles, the marginal effect of reduced dyspepsia can represent savings to a managed care provider. Specifically, the estimates detailed here indicate that a reduction in dyspepsia from $20 \%$ to $8 \%$ results in an estimated marginal cost savings to the insurer of $\$ 14$ per treated patient with etodolac ER versus conventional etodolac. In a health care environment in which outcomes and costeffectiveness go hand-in-hand, it is important to seek treatment options that achieve both goals. 


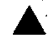

References

1. Boh LE. Osteoarthritis. In: DiPiro JT, Talbert RL, Hayes PE, et al., eds. Pharmacotherapy: A Pathophysiologic Approach. 2nd ed. Norwalk, CT: Appleton \& Lange; 1993: 1330-42.

2. Peyron JG. Osteoarthritis: the epidemiologic viewpoint. Clin Orthop 1986; 213: 13-19.

3. Lawrence RC, Hochberg MC, Kelsey JL, et al. Estimates of the prevalence of selected arthritic and musculoskeletal diseases in the United States. J Rheumatol 1989; 16: 427-41.

4. Hutton CW. Treatment, pain, and epidemiology of osteoarthritis. Current Opin Rheumatol 1990; 2: $765-69$.

5. Gibson $T$. Nonsteroidal anti-inflammatory drugsanother look. Br J Rheumatol 1989; 27: 87-90. 6. Carson JL, Strom BL, Soper KA, West SL, Morse $\mathrm{ML}$. The association of nonsteroidal anti-inflammatory drugs with upper gastrointestinal bleeding Arch Intern Med 1987; 147: 85-88.

7. The top 200 drugs of 1994. Am Druggist 1995; 211: 20-24.

8. Waterworth RF, Petrie JP. Double-blind comparative study of etodolac and piroxicam in the treatment of patients with osteoarthritis of the knee. Adv Ther 1992; 9: 240-49.

9. Paulsen GA, Baigun $S$, deFigueiredo JG, deFreitas GC. Efficacy and tolerability comparison of etodolac and piroxicam in the treatment of patients with osteoarthritis of the knee. Curr Med Res Opin 1991; 12: 401-12.

10. Pena M, Lizarazo H. Double-blind comparison of etodolac and naproxen in patients with osteoarthritis of the knee. Acta Ther 1991; 17: 19-34. 11. Karbowski A. Double-blind, parallel comparison of etodolac and indomethacin in patients with osteoarthritis of the knee. Curr Res Opin 1991; 12: 309-17.

12. Brasseur JP, Raeman F, Franchimont P. Doubleblind parallel comparison of etodolac and diclofenac SR in patients with osteoarthritis of the knee. Acta Ther 1991; 17: 345-54.

13. Bloom BS. Costs of treating arthritis and NSAID-related gastrointestinal side effects. Aliment Pharmacol Ther 1988; 2(suppl): 131-39. 14. Larkai EN, Smith JL, Lidsky MD, et al. Gastroduodenal mucosa and dyspeptic symptoms in arthritic patients during chronic nonsteroidal antiinflammatory drug use. Am J Gastroenterol 1987; 82: 1153-58.

15. Lanza FL. Gastrointestinal toxicity of newer NSAIDs. Am J Gastroenterol 1993; 88: 1318-23. 16. Ward JR. Nonsteroidal (nonsalicylate) antiinflammatory drugs. In: Roth SH, ed. Rheumatic Therapeutics. New York, NY: McGraw Hill International Book Co.; 1985: 383-96. 17. Jallad NS, 'Sanda M, Salom IL, et al. Gastrointestinal blood loss in arthritic patients receiving chronic dosing with etodolac and piroxicam. Am J Med Sci 1986; 292: 272-76.

18. Arnold JD, Salom IL, Berger AE, et al. Comparison of gastrointestinal microbleeding associated with use of etodolac, ibuprofen, indomethacin, and naproxen in normal subjects. Curr Ther Res 1985; 37: 730-38.

19. Lanza F, Rack MF, Lynn M, Wolf J, Sanda M An endoscopic comparison of the effects of etodolac, indomethacin, ibuprofen, naproxen, and placebo on the gastrointestinal mucosa. J Rheumatol 1987 ; $14: 338-41$

20. Bianchi Porro G, Caruso I, Petrillo M, et al. A double-blind gastroscopic evaluation of the effects of etodolac and naproxen on the gastrointestinal mucosa of rheumatic patients. J Intern Med 1987; 229: 5-8

21. Lanza FL, Arnold JD. Etodolac, a new nonsteroidal anti-inflammatory drug: gastrointestinal microbleeding and endoscopic studies. Clin Rheumatol 1989; 8(suppl 1): 5-15.

22. Leese P. Comparison of the effects of etodolac SR and naproxen on gastrointestinal blood loss. Curr Med Res \& Opinion 1992; 13(1): 13-20. 23. Perpignano G, Bogliolo A, Puccetti L. Doubleblind comparison of the efficacy and safety of etodolac SR $600 \mathrm{mg}$ u.i.d. and of tenoxicam 20 mg u.i.d. in elderly patients with osteoarthritis of the hip and of the knee. Intl J of Clinical Pharmacology Res 1994; 14(5-6): 203-16. 24. Edelson JT, Tosteson ANA, Sax P. Cost effectiveness of misoprostol for prophylaxis against nonsteroidal anti-inflammatory drug-induced gastrointestinal tract bleeding. JAMA 1990; 264: 41-47. 25. Singh G, Ramey DR, Morfeld D, et al. Gastrointestinal tract complications of nonsteroidal anti-inflammatory drug treatment in rheumatoid arthritis. Arch Intern Med 1996; 156: 1530-36.

26. Kahn FM, Williams PI. Double-blind comparison of etodolac SR and diclofenac SR in the treatment of patients with degenerative joint disease of the knee. Curr Med Res Opin 1992; 12: 615-26. 27. Dreiser RL. A comparison of the efficacy of etodolac SR (Lodine (BSR) and etodolac (Lodine ${ }^{\circledR}$ ) in patients with rheumatoid arthritis or osteoarthritis. Rheumatol Int 1993; 13: S13-S8. 28. Anonymous. Med Ad News, 1996 (May): 19 29. Walker AM, Chan KA, Yood RA. Patterns of Interchange in the Dispensing of Non-Steroidal Antilnflammatory Drugs. J Clin Epidemiol 1992; 45(2): 187-95 